\title{
In-situ Observation of Incompressible Mott-Insulating Domains of Ultracold Atomic Gases
}

\author{
Nathan Gemelke, Xibo Zhang, Chen-Lung Hung, and Cheng Chin \\ The James Franck Institute and Department of Physics, \\ University of Chicago, Chicago, IL 60637, USA
}

(Dated: April 9, 2009)

\begin{abstract}
We present a direct measurement of the density profile of a two-dimensional Mott Insulator formed by ultracold atoms in an optical lattice. High resolution absorption imaging is used to probe the "wedding-cake" structure of a trapped gas as it crosses the boundary from a unit-filled Mott insulating phase to the superfluid phase at finite temperature. Detailed analysis of images yields measurements of temperature and local compressibility; for the latter we observe a strong suppression deep in the Mott-insulating phase, which is recovered for the superfluid and normal phases. Furthermore, we measure spatially resolved fluctuations in the local density, showing a suppression of fluctuations in the insulator. Results are consistent with the fluctuation-dissipation theorem for insulator, superfluid and normal gas.
\end{abstract}

PACS numbers: 73.43.Nq,03.75.Kk,05.30.Jp,03.75.Lm

The observation of the Superfluid (SF) to Mottinsulator (MI) phase transition of ultracold atoms in optical lattices [1] marked an experimental breakthrough in the study of many-body physics in an atomic system. This development provided the first tangible example of a quantum phase transition (one that occurs even at zero temperature) and suggested a highly correlated and gapped Mott insulating state of a Bose gas, which persists down to zero temperature without Bosecondensation and macroscopic phase coherence. Since its theoretical inception [2, 3, 4], two of the most celebrated properties of the bosonic Mott insulator have been its incompressibility and suppression of local density fluctuations [5], induced by enhanced inter-particle interaction and reduced mobility in optical lattices. The result for a trapped atom gas, where the local chemical potential varies in space, is the remarkable "wedding-cake" density profile, where successive MI domains are manifest as plateaus of constant density. Related phenomena have been studied through the coherence [1, ㅎ], transport [1, 7], noise correlations [8], and number variance [9, 10], but direct observation of the incompressibility has proven difficult due to the inhomogeneous nature of all experiments to date, and to the technical difficulty of spatially resolved measurements. Innovative experimental efforts have yielded evidence [11, 12] that these plateaus exist, though none has directly observed this effect by imaging a single physical system in situ.

We report studies based on direct in-situ imaging of an atomic MI. By loading a degenerate Bose gas of cesium133 atoms into a thin layer of a two-dimensional optical lattice potential, and adiabatically increasing the optical lattice depth, we observe the emergence of an extremely flat density near the center of the cloud, which corresponds to a MI phase with accurately one atom-per-site. From density profiles, we extract important thermodynamic and statistical information, confirming the incompressibility and reduction of density fluctuations in the MI as described by the fluctuation-dissipation theorem.
The single layer, two-dimensional (2D) optical lattice is formed by two pairs of counter-propagating laser beams derived from a Yb fiber laser at wavelength $\lambda=1064 \mathrm{~nm}$. The pairs are oriented orthogonally on the horizontal $(x-y)$ plane, forming a square lattice with site spacing $d=\lambda / 2=0.532 \mu \mathrm{m}$. A weak harmonic potential of $V_{H}=m\left(\omega_{x}^{2} x^{2}+\omega_{y}^{2} y^{2}\right) / 2$ localizes the sample, where $m$ is the cesium mass, and the geometric mean of the trap frequencies is $\omega_{r}=\sqrt{\omega_{x} \omega_{y}}=2 \pi \times 9.5\left(1+V / 82 E_{R}\right) \mathrm{Hz}$; we have included a weak dependence on the lattice depth $V\left(E_{R}=h 1.3 \mathrm{kHz}\right.$ is the lattice recoil energy and $h$ is Planck's constant.) Vertical confinement is provided by an additional vertical optical lattice with a site spacing $4 \mu \mathrm{m}$, formed by two beams intersecting at an angle of $15^{\circ}$, confining atoms to an oscillator length $a_{z}=0.30 \mu \mathrm{m}$. The sample is loaded into a single site of the vertical lattice, kept deep to prevent vertical tunneling. Tunneling in the horizontal 2D lattice is controlled by varying the lattice depth $V$ [1]. Details on preparation of the atomic sample can be found in the supporting material and Ref. [13].

We obtain a top view of the sample using absorption imaging, directly revealing the atomic surface density $n(x, y)$ on the horizontal plane. The imaging resolution is $3 \sim 4 \mu \mathrm{m}$, and magnification such that one imaging pixel corresponds to an area of $(2 \mu \mathrm{m})^{2}$ on the object plane. Conveniently, unit filling in a $2 \mathrm{D}$ optical lattice has a moderate optical density of $O D=O(1)$ on resonance.

The superflulid-to-Mott insulator (SF-MI) transition of ultracold atoms in an optical lattice is described by the Bose-Hubbard model, characterized by on-site interaction $U$ and the tunneling $J$ [4]. In 2D optical lattices, superfluid is converted into a MI when $U / J$ exceeds 16 6, 14]. Here, the SF-MI phase transition can be induced by either increasing the lattice potential $V$ [1, 6, 12] or the atomic scattering length $a$ via a magnetically-tuned Feshbach resonance [15], together providing complete, independent control of $U$ and $J$.

Atomic density profiles in the lattice are shown in 


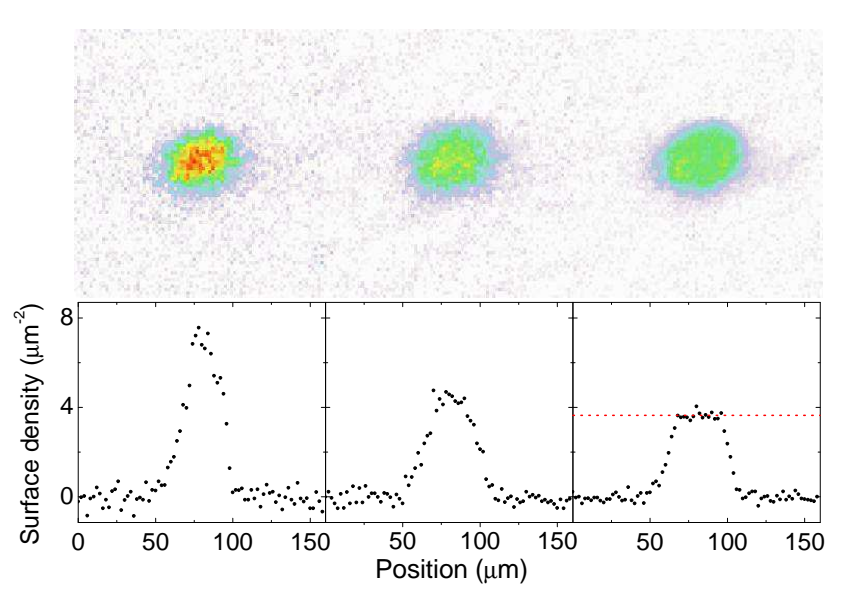

FIG. 1: False color density profiles and line cuts of $N=7500$ ultracold cesium atoms at scattering length $a=310 a_{B}$ in $2 \mathrm{D}$ optical lattices. (A) Superfluid regime (shallow lattice $V=$ $0.14 E_{R}$ ), (B) Phase transition regime (medium lattice depth $V=7.2 E_{R}$ ), and (C) Mott insulator regime (deep lattice $V=38 E_{R}$ ) The red dashed line indicates the expected density for one atom-per-site. Line cuts pass through the center of the sample.

Fig. 1. For weak lattice depths (superfluid regime), the density profiles are bell-shape, with negative curvature at the center (Fig. 1A A), indicating a finite, positive compressibility dictated by the interaction coupling constant (discussed below.) In sufficiently deep lattices, we observe a flattened density at the center of the sample (Fig. 1B, C), indicating development of a Mott insulating phase with one particle per lattice site. This density plateau, an important feature of the MI phase, arises from incompressibility.

A primary check on the MI is to compare the measured density in the plateau to that corresponding to one atomper-site, given by MI physics as a "standard candle" of atomic density. Using the known scattering cross-section, correcting for saturation effects (see Methods), we determine the plateau density to be $n=3.5(3) / \mu \mathrm{m}^{2}$, in agreement with the expected value $1 / d^{2}=3.53 / \mu \mathrm{m}^{2}$.

To distinguish a MI from superfluid or normal gas, we histogram the occurrence of pixels $h(n)$ in the images corresponding to a density $n$ with a bin size of $\Delta n \ll n$. The MI plateau, containing a large number of pixels with similar atomic density, appears as a peak at $n=1 / d^{2}$ (Fig. 22A). In general, the occurrence of a particular density $n$ can be regarded as the rate at which local chemical potential changes with density, multiplied by the number of pixels $w(\mu) \Delta \mu$ corresponding to a chemical potential between $\mu$ and $\mu+\Delta \mu$. The occurrence at density $n$ is then $h(n)=\Delta n w(\mu) \Delta \mu / \Delta n \approx \Delta n w(\mu) \kappa^{-1}$, where $\kappa=\partial n / \partial \mu$ is the local compressibility [16]. In a harmonic trap, $w(\mu)=2 \pi / m d^{2} \omega_{r}^{2}$ is constant, and the histogram is a particularly useful tool to distinguish different phases. For a pure BEC in the Thomas-Fermi limit, the compressibility is constant to the maximally-allowed density $n_{p k}$, and results in a constant $h(n)$ for $n \leq n_{p k}$

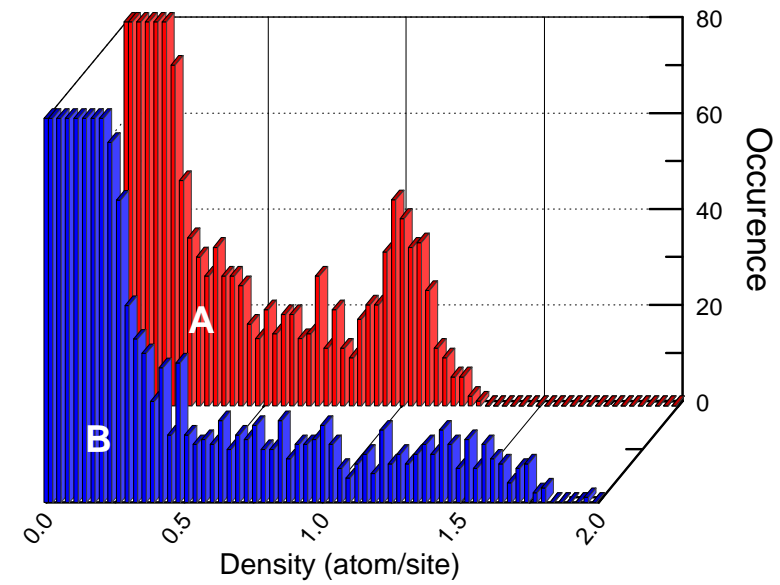

FIG. 2: Histograms of density profiles in the MI regime (A, $\left.V=38 E_{R}, a=460 a_{B}\right)$ and the superfluid regime (B, $V=$ $0.5 E_{R}, a=460 a_{B}$.) The histograms are based on an average of three density images. The bin size is $\Delta n=0.03$.

(see Fig. $2 \mathrm{~B}$ for $0.5 / d^{2}<n<1.5 / d^{2}$ ). For the MI, the density is insensitive to chemical potential in a narrow range near $n=1 / d^{2}$, indicating a vanishing compressibility, and thus a sharp histogram peak at $n=1 / d^{2}$. The peak's presence in Fig. 2A is thus directly related to the incompressibility in the Mott phase. Finally, the compressibility of a normal (ideal) gas is proportional to its density, thus $h(n) \propto 1 / n$, leading to the strong upturn at low densities in Fig. 2A,B for both regimes.

Much more information can be obtained from the density profiles, as recently suggested in Ref. 17]. For example, the compressibility in a two-dimensional cylindrically symmetric trap can be written $\kappa=\partial n / \partial \mu=$ $-n^{\prime}(r) /\left(r m \omega_{r}^{2}\right)$, where we have assumed the local density approximation, and that the chemical potential depends on the trapping potential $\mu=\mu_{0}-V_{H}(r)$. For a BEC in the Thomas-Fermi regime, the compressibility is a positive and constant $\kappa_{B E C}=1 / g$, where $g=\sqrt{8 \pi} a \hbar^{2} / m a_{z}$ is the $(2 \mathrm{D})$ interaction parameter [18]. We can thereby relate the measured compressibility to that of a BEC as

$$
\frac{\kappa}{\kappa_{B E C}}=-\left(\frac{2}{\pi}\right)^{7 / 2} \frac{n^{\prime}(r)}{r d^{-4}} \frac{a}{a_{z}}\left(\frac{E_{R}}{\hbar \omega_{r}}\right)^{2} .
$$

We evaluate $\kappa$ from azimuthally averaged density profiles (Fig. 3 A). Eccentricity of the trap is corrected by rescaling the principal axes. Due to the singular nature of $n^{\prime}(r) / r$ near the center, we evaluate $\kappa$ there by fitting $n(r)$ to a quadratic, $n(r)=n(0)-\alpha r^{2}$. The curvature then gives the compressibility as $\kappa(0)=2 \alpha / m \omega_{r}^{2}$, for which we obtain $\kappa / \kappa_{B E C}=0.4(1)$ in a weak lattice and $\kappa / \kappa_{B E C}=0.006(6)$ in a strong lattice (See Fig. 3). In the weak lattice (SF regime), the finite and constant compressibility at the center agrees with expectation for the superfluid phase, though lower than expected, which we attribute to finite temperature and calibration of trap parameters. The finite temperature is also clear in the 


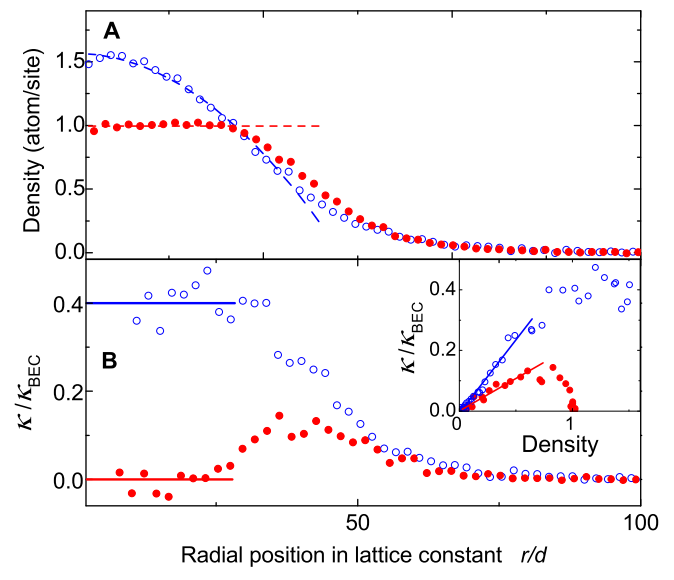

FIG. 3: (A) Radially averaged profiles (3 images) in the superfluid (blue open circles: $V=0.25 E_{R}$ ) and MI (red circles: $\left.V=38 E_{R}\right)$ regimes. Particle number and scattering length are $N=8100$ and $a=460 a_{B}$, respectively. A quadratic fit to the center portion of the sample extracts the curvature near $r=0$. (B) Normalized compressibilities derived from (A) using Eq. (1) in the superfluid (blue open circles) and MI (red circles) regimes. The horizontal lines indicate the compressibility near $r=0$, estimated from the quadratic fits in (A). The rising compressibility at $r=40 d$ marks the MI boundary. The inset shows the dependence of compressibility on atomic density. The linear dependence at low densities is fit by the solid lines.

exponential tail of the density profile and the compressibility, from which we derive the temperature 11(2) nK in the superfluid regime and 24(4) nK in the MI regime.

In a deep lattice (MI regime), we observe a strong reduction of the compressibility in the trap center, below that in the superfluid phase for the weak lattice, strongly supporting the emergence of a MI phase at the center of the sample. Away from center, $\kappa$ suddenly increases at $r=25 d$, then decreases for $r>50 d$. The exponential decay is again consistent with a normal gas. Between MI and normal gas $(25 d<r<50 d)$, a more detailed measurement and model of compressibility would be necessary to identify the local phase.

Incompressibility necessarily implies a low particle number fluctuation; this relationship, a result of the fluctuation-dissipation theorem (FDT) (see e.g. [19, 20]), takes the form

$$
\delta n^{2}=\kappa k_{B} T
$$

Resolved in-situ imaging provides an enticing opportunity to measure fluctuations of the local density [5, 21], and thus check the validity of the FDT. We measured fluctuations by recording multiple absorption images, calculating the variance of density measured in each pixel (each collects signals from a patch of $(2 \mu \mathrm{m} / d)^{2} \approx 14$ lattice sites). Fig. 4 shows the recorded fluctuations, where

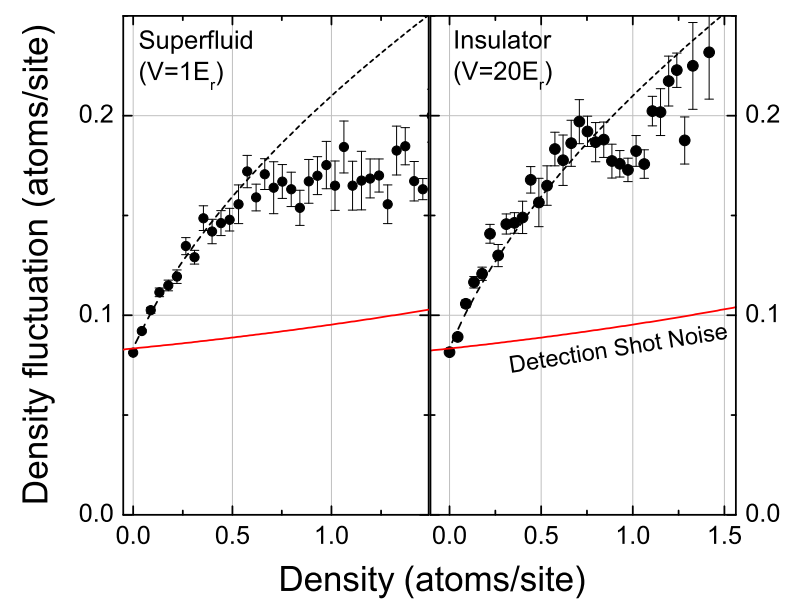

FIG. 4: The fluctuation of local density extracted from a set of twelve absorption images in the deep (MI) and weak (SF) lattice regimes. The insulator and superfluid show a pronounced difference at the density of one atom-per-site, where the insulator's fluctuation is suppressed by its incompressibility. In the superfluid phase, constant compressibility initiates a flattening of fluctuations with density. At low densities, in both regimes, the fluctuation shows a characteristic $\sqrt{n}$ dependence. The dashed line shows best fit $\sqrt{n}$ dependence for the normal gas. The total number of atoms was $N=8300$ (SF) and $N=9600(\mathrm{MI})$ with $a=310 a_{B}$ for both sets.

pixels are binned according to their mean density. Fluctuations consist of detection (photo-electron shot) noise and thermal and quantum atomic density fluctuations. Detection shot noise can be well-calibrated and modeled by analyzing portions of the images with low density; extension to higher optical depth (density) shows the weak dependence illustrated (Fig. 4).

Above the detection noise, density fluctuations (see Fig. (4) show a strong qualitative agreement with the compressibility presented in Figure 3 as expected from the FDT. For example, the Mott-phase shows a strong suppression of fluctuations at the density of one atomper-site. The superfluid regime lacks this feature, instead showing a pronounced flattening as the sample transitions from normal gas to superfluid, as expected from the constant compressibility in the superfluid phase (Figure 3. inset). Finally, at low density, the normal gas shows a temperature-independent fluctuation of $\delta n=\gamma \sqrt{n}$, which can be anticipated from the inset of Figure 3 and agrees with the FDT. The coefficient $\gamma$ is roughly consistent with the FDT, and measured imaging resolution (see methods).

Clearly, in situ imaging of the Mott insulator is a powerful new tool to investigate new quantum phases of cold atoms in optical lattices. From the density profiles, not only can one observe the density plateau, incompressibility and reduction of fluctuations in the Mott insulating 
phase, but also demonstrate a qualitative validation of the fluctuation-dissipation theorem. Relatively modest extension of this work holds new promise for studying the role of quantum fluctuations, correlation and thermodynamics near a quantum phase transition.

We thank T.L. Ho, R. Scalettar, E. Mueller, and R.
Hulet for helpful discussion. This work is supported by NSF No. PHY-0747907, NSF-MRSEC DMR-0213745, and ARO No. W911NF0710576 with funds from the DARPA OLE Program. N.G. acknowledges support from the Grainger Foundation.

[1] M. Greiner, O. Mandel, T. Esslinger, T. W. Hansch, and I. Bloch, Nature 415, 39 (2002).

[2] M. I. Kaganov and A. V. Chubukov, Uspekhi Fizicheskikh Nauk 153, 537 (1987).

[3] M. P. A. Fisher, P. B. Weichman, G. Grinstein, and D. Fisher, Physical Review B 40, 546 (1989).

[4] D. Jaksch, C. Bruder, J. I. Cirac, C. W. Gardiner, and P. Zoller, Physical Review Letters 81, 3108 (1998).

[5] B. Capogrosso-Sansone, N. V. Prokof'ev, and B. V. Svistunov, Physical Review A 75, 013619 (2007).

[6] I. B. Spielman, W. D. Phillips, and J. V. Porto, Phys Rev Lett 100, 120402 (2008).

[7] M. Kohl, H. Moritz, T. Stoferle, C. Schori, and T. Esslinger, Journal of Low Temperature Physics 138, 635 (2005).

[8] S. Folling, F. Gerbier, A. Widera, O. Mandel, T. Gericke, and I. Bloch, Nature 434, 481 (2005).

[9] M. Greiner, O. Mandel, T. W. Hansch, and I. Bloch, Nature 419, 51 (2002).

[10] F. Gerbier, S. Folling, A. Widera, O. Mandel, and I. Bloch, Physical Review Letters 96, 090401 (2006).

[11] S. Folling, A. Widera, T. Muller, F. Gerbier, and I. Bloch, Phys Rev Lett 97, 060403 (2006).

[12] G. K. Campbell, J. Mun, M. Boyd, P. Medley, A. E. Leanhardt, L. G. Marcassa, D. E. Pritchard, and W. Ketterle, Science 313, 649 (2006).

[13] C. L. Hung, X. B. Zhang, N. Gemelke, and C. Chin, Physical Review A 78, 011604 (2008).

[14] I. B. Spielman, W. D. Phillips, and J. V. Porto, Physical Review Letters 98, 080404 (2007).

[15] C. Chin, R. Grimm, P. Julienne, and E. Tiesinga, arXiv.org:0812.1496 (2008).

[16] M. Rigol, G. G. Batrouni, V. Rousseau, and R. T. Scalettar, arXiv:0811.2219 (2008).

[17] T.-L. Ho and Q. Zhou, arXiv:0901.0018v1 (2008).

[18] B. Tanatar, A. Minguzzi, P. Vignolo, and M. P. Tosi, Physics Letters A 302, PII S0375 (2002).

[19] G. G. Batrouni, V. Rousseau, R. T. Scalettar, M. Rigol, A. Muramatsu, P. J. H. Denteneer, and M. Troyer, Physical Review Letters 89, 117203 (2002).

[20] K. Huang, Statistical mechanics (Wiley, New York, 1963).

[21] J. Esteve, J. B. Trebbia, T. Schumm, A. Aspect, C. I. Westbrook, and I. Bouchoule, Physical Review Letters 96, 090401 (2006). 


\section{Supporting Material}

\section{Preparation of BEC in a thin 2D optical lattice}

The ${ }^{133} \mathrm{Cs}$ BEC is formed in a crossed-beam dipole trap by an efficient evaporative cooling method [1]. The dipole trap consists of three beams on the horizontal plane: two orthogonal beams at the wavelength of $1064 \mathrm{~nm}$ (Yb fiber laser, YLR-20-1064-LP-SF, IPG), focused to $1 / e^{2}$ radii of $350 \mu \mathrm{m}$, and one $\mathrm{CO}_{2}$ laser beam (not shown in Fig 5) at the wavelength of $10.6 \mu \mathrm{m}$ (Gem-Select 100, Coherent), focused to a vertical $1 / e^{2}$ radius of $70 \mu \mathrm{m}$ and horizontal of $2 \mathrm{~mm}$ (see Fig. 5). The $\mathrm{CO}_{2}$ beam intersects the $\mathrm{Yb}$ laser beams at an angle of $45^{\circ}$ and provides an enhanced vertical confinement to support the atoms against gravity. With $N=10^{4}$ atoms in a pure condensate, the Thomas-Fermi radii of the condensate are $\left(r_{x}, r_{y}, r_{z}\right)=(23,14,3.6) \mu \mathrm{m}$.

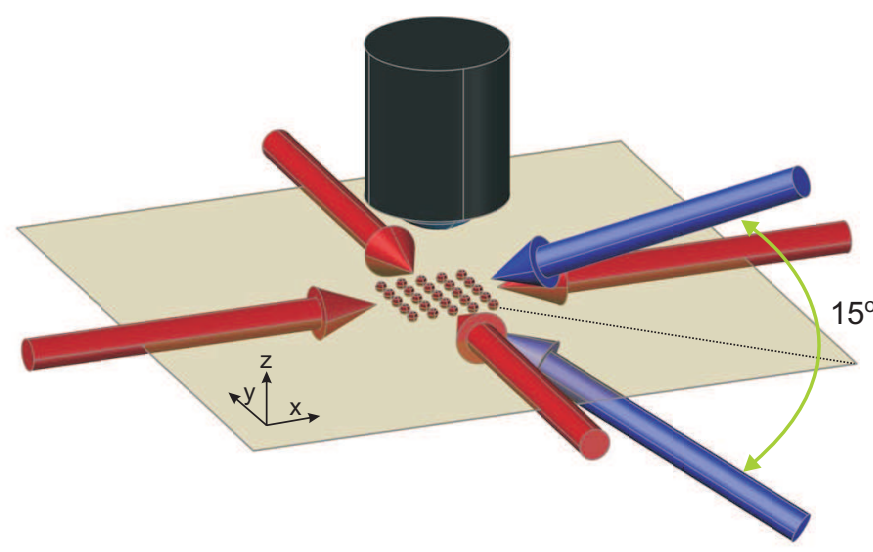

FIG. 5: Experimental apparatus used to investigate quantum phases in two-dimensional optical lattices. Two pairs of counterpropagating beams (red) form a 2D square optical lattice, into which a cesium Bose-condensate is loaded. Atoms are confined in the vertical direction by loading into a single site of a separate one-dimensional lattice in the vertical direction, formed by two beams (blue) intersecting at a small angle. A high numerical aperture imaging lens images the shadow (absorption) cast by the atoms illuminated by imaging light propagating vertically.

After a pure BEC is obtained, the sample is compressed vertically by introducing a vertical lattice, formed by two laser beams (Innolight Mephisto) inclined at $+7.5^{\circ}$ and $-7.5^{\circ}$ relative to the horizontal plane. The vertical lattice has a spacing of $4 \mu \mathrm{m}$ and, together with the crossed dipole trap, forms an array of 2D oblate "pancake" potentials.

In order to load the condensate into a single pancake trap, we first ramp the magnetic field to $17.2 \mathrm{G}$ in $400 \mathrm{~ms}$, reducing the s-wave scattering length to $a<10 a_{B}$, and then turn on the vertical lattice in $100 \mathrm{~ms}$. Atomic population in other lattice sites, if any, can be identified by observing an interference pattern in time-of-flight images taken from the side. For this work, we observe a sufficiently weak interference pattern contrast to conclude $>98 \%$ of the atoms are in a single pancake trap. After the vertical lattice is fully turned on, the $\mathrm{CO}_{2}$ laser intensity is ramped to zero in $100 \mathrm{~ms}$ and the scattering length ramped to a desired value by tuning the external magnetic field.

The 2D lattice potential in the horizontal ( $x$ - and $y$-) directions is formed by introducing retro-reflections of the $1064 \mathrm{~nm}$ dipole trap beams. A continuous evolution from a pure dipole trap (with zero retro-reflection) to a $2 \mathrm{D}$ optical lattice (with significant retro-reflection) is achieved by passing each dipole trap beam (after it passes through the atomic cloud once) through two acousto-optic modulators (AOMs) controlled by the same radio-frequency (rf) source, then off a retroreflection mirror. The AOMs induce an overall zero frequency shift, but permit a dynamic control of the retroreflection intensity over six orders of magnitude. Onsite interaction energy $U$ and tunneling rate $J$ are evaluated from the measurements of the lattice vibration frequencies and the band structure calculation.

\section{Calibration of atomic surface density}

By varying the intensity of the imaging beam, we measure the optical depth in the density plateau using $O D=\ln \left(M_{0} / M\right)$, where $M$ is the number of photons collected by a CCD pixel in the presence of the atoms and $M_{0}$ is that without the atoms. The optical depth in the plateau is extracted from a fit to the peak in the histogram. We then fit the variation of peak optical depth assuming $O D=n \sigma /\left(1+M_{0} / M_{\text {sat }}\right)$ to determine the depth in the zero intensity limit $M_{0} \rightarrow 0$, and thus the surface density of the sample. Here, $\sigma=0.347 \mu \mathrm{m}^{2}$ is the known cesium atom-photon cross-section while the fit parameter $M_{\text {sat }}$ represents the photon number on a CCD pixel at the atomic saturation intensity.

\section{Fluctuation of atomic density}

The fluctuations in the absorption images are estimated by taking the average of 11 images under the same experimental procedure, and calculating the mean and variance of optical depth measured at each CCD pixel. Fluctuations are presumed to arise from optical shot noise, thermal atomic fluctuation, and long lengthscale variations arising from total atom number fluctuation. The optical shot noise is calibrated by examining regions with negligible atomic density, and extended to higher optical depth using $\delta O D_{o s} \propto \sqrt{1+e^{O D}}$. For the thermal cloud, with density $n<0.3$ atoms/site, the fluctuationdissipation theorem predicts $\delta N_{a}=\sqrt{N_{a}}$, with $N_{a}$ the number of atoms measured in a given region. This result should be valid for a region significantly larger than 
the correlation length, which we expect for the normal gas to be on order of the deBroglie thermal wavelength, expected to be $<1.5 \mu \mathrm{m}$ for our sample. Though each imaging pixel corresponds to an area in the object plane consisting of 14 sites, imperfect imaging resolution is expected to effectively average away a certain fraction of the total fluctuation. This effect can be calculated, assuming statistical independence for each site, by summing the weight $w_{i, j}$ of a resolution-limited spot falling within a given pixel $\mathrm{j}$ for each lattice site $\mathrm{i}$, giving a variance reduced by $\sum_{i} w_{i, j}^{2}$. The result for our parameters is a reduction to $\delta n=\gamma \sqrt{n}$, with $\gamma \sim 0.11(1)$. This should be compared with the fraction of the total fluctuation shown in Fig. 4 corresponding to thermal fluctuations in the superfluid regime. To make this comparison, we reject global fluctuations associated with variation of the total atom number by subtracting the variance we calculate after first applying a resolution-spoiling gaussian blur to the images from the variance without modification. We find, for the remaining high spatial-frequency fluctuations, a best fit to $\gamma$ of $0.15(2)$, using a gaussian blur $1 / e^{2}$ radius of $r_{b}=14 \mu \mathrm{m}$ to remove global variations (the result varies within stated error for blur radii $\left.7 \mu \mathrm{m}<r_{b}<28 \mu \mathrm{m}\right)$. The remaining discrepancy is likely due to calibration of imaging resolution, and possibly the effect of a nonnegligible correlation length.

\section{References and Notes}

1. C. L. Hung, X. B. Zhang, N. Gemelke, C. Chin, Physical Review A, 78, 011604 (2008). 